

\title{
Characterization of a nuclear pore protein sheds light on the roles and composition of the Toxoplasma gondii nuclear pore complex
}

Flavie Courjol, Thomas Mouveaux, Kevin Lesage, Jean-Michel Saliou, Elisabeth Werkmeister, Maurine Bonabaud, Marine Rohmer, Christian

Slomianny, Franck Lafont, Mathieu Gissot

\section{To cite this version:}

Flavie Courjol, Thomas Mouveaux, Kevin Lesage, Jean-Michel Saliou, Elisabeth Werkmeister, et al.. Characterization of a nuclear pore protein sheds light on the roles and composition of the Toxoplasma gondii nuclear pore complex. Cellular and Molecular Life Sciences, 2017, 74 (11), pp.2107-2125. 10.1007/s00018-017-2459-3 . hal-02106431

\section{HAL Id: hal-02106431 \\ https://hal.science/hal-02106431}

Submitted on 23 Apr 2019

HAL is a multi-disciplinary open access archive for the deposit and dissemination of scientific research documents, whether they are published or not. The documents may come from teaching and research institutions in France or abroad, or from public or private research centers.
L'archive ouverte pluridisciplinaire HAL, est destinée au dépôt et à la diffusion de documents scientifiques de niveau recherche, publiés ou non, émanant des établissements d'enseignement et de recherche français ou étrangers, des laboratoires publics ou privés. 
Flavie Courjol ${ }^{1}$, Thomas Mouveaux ${ }^{1}$, Kevin Lesage ${ }^{1}$, Jean-Michel Saliou ${ }^{1}$, Elisabeth Werkmeister ${ }^{1}$, Maurine Bonabaud $^{2}$, Marine Rohmer ${ }^{2}$, Christian Slomianny ${ }^{3}$, Franck Lafont ${ }^{1}$ and Mathieu Gissot ${ }^{1, *}$.

1: Univ. Lille, CNRS, Inserm, CHU Lille, Institut Pasteur de Lille, U1019 - UMR 8204 - CIIL - Centre d'Infection et d'Immunité de Lille, F-59000 Lille, France.

11 Montpellier Cedex 5, France.

3 : Laboratory of Cell Physiology, INSERM U 1003, Université Lille Nord de France, Villeneuve d'Ascq, France

* Corresponding author: mathieu.gissot@pasteur-lille.fr

\section{Acknowledgements}

The authors would like to thank Dr. Valerie Doye for helpful discussions and Dr. R. Walker for critically reading the manuscript. We also thank Ludovic Huot for checking the integrity of RNA samples, Etienne Dewailly for electronic microscopy, Antonino Bongiovanni for his help with microscopy data analyses, and Quentin Deveuve for phylogenetic tree recommendations. The authors also thank the BioImaging Center Lille for access to instruments. This work was supported by Centre National de la Recherche Scientifique (CNRS), Institut National de la Santé et de la Recherche Médicale (INSERM), grants from the French National Research Agency (ANR) [grant number ANR-13-JSV3-0006-01 to MG and ANR-11-LABX-0024 to Plateforme Protéomique et Peptides Modifiés (P3M)], the Fonds Européen de Développement Economique Régionale (13003300-42405 Labex Parafrap to P3M) and the Métropole Européenne de Lille (MEL). 
The nuclear pore is a key structure in eukaryotes regulating nuclear-cytoplasmic transport as well as a wide range of cellular processes. Here, we report the characterization of the first Toxoplasma gondii nuclear pore protein, named TgNup302, which appears to be the orthologue of the mammalian Nup98-96 protein. We produced a conditional knock-down mutant that expresses TgNup302 under the control of an inducible tetracycline-regulated promoter. Under ATc treatment, a substantial decrease of TgNup302 protein in iKD parasites was observed, causing a delay in parasite proliferation. Moreover, the nuclear protein TgENO2 was trapped in the cytoplasm of ATc-treated mutants, suggesting that TgNup302 is involved in nuclear transport. Fluorescence in situ hybridization revealed that TgNup302 is essential for 18S RNA export from the nucleus to the cytoplasm, while global mRNA export remains unchanged. Using an affinity tag purification combined with mass spectrometry, we identified additional components of the nuclear pore complex, including proteins potentially interacting with chromatin. Furthermore, reverse immunoprecipitation confirmed their interaction with $\operatorname{TgNup} 302$, and structured illuminated microscopy confirmed the NPC localization of some of the TgNup302-interacting proteins. Intriguingly, facilitates chromatin transcription components were identified, suggesting the existence of an NPC-chromatin interaction in T. gondii. Identification of TgNup302-interacting proteins also provides the first glimpse at the NPC structure in Apicomplexa, suggesting a structural conservation of the NPC components between distant eukaryotes.

49 Keywords: Toxoplasma gondii, nuclear pore complex, nucleoporins, gene expression, apicomplexa

\section{Abbreviations:}

51 ATc: AnhydroTetraCycline

52 Co-IP: Co- ImmunoPrecipitation

53 FACT: Facilitates Chromatin Transcription

54 FISH: Fluorescence in situ Hybridization

55 IFA: ImmunoFluorescence Assay

56 iKD: conditional Knock-Down

57 NPC: Nuclear Pore Complex

58 NUP: Nucleoporin

59 RNA Seq: RNA Sequencing

60 Sg RNA: Single guide RNA 
61 SIM: Structured Illumination Microscopy

62 Tg: Toxoplasma gondii

63

64

65

66

67

68

69

70

71

72

73 
Toxoplasma gondii is a unicellular eukaryotic pathogen. It belongs to the apicomplexan phylum, which encompasses some of the deadliest pathogens of medical and veterinary importance, including Plasmodium (the cause of malaria), Cryptosporidium (responsible for cryptosporidiosis) and Eimeria (coccidiosis). T. gondii is an obligate intracellular parasite that leads to the development of focal central nervous system infections in patients with HIV/AIDS. In addition, Toxoplasma is also a clinically important opportunistic pathogen that can cause birth defects in the offspring of newly infected mothers. The life cycle of $T$. gondii is complex, with multiple differentiation steps that are critical to parasite survival in human and feline hosts [1]. Although gene expression is tightly controlled in Apicomplexa, which is particularly evident during the cell cycle [2] [3], the molecular mechanisms underlying its regulation are poorly understood. Initial studies suggest that histone modifications and chromatin remodeling have an important role in chromatin structure for gene regulation [4] [5]. Eukaryotic nuclei are enclosed by the double-membrane nuclear envelope (NE), which is perforated by large protein structures termed nuclear pore complexes (NPCs), allowing a controlled bidirectional nucleocytoplasmic transport of macromolecules [6]. In eukaryotes, the organization and composition of NPCs has remained conserved throughout evolution, and they play an important role in other biological processes, such as chromosomal segregation mechanisms, mitotic spindle formation, transcription activation and cytokinesis [7]. Each NPC is composed of multiple copies of approximately 30 different proteins known as nucleoporins (NUPs), for which the composition and structure have been largely characterized in Saccharomyces cerevisiae [8], mammals [9] [10] and Trypanosoma brucei $[11,12]$. Strikingly, the description of the putative components of the NPC in T. brucei led to the discovery of a conserved protein arrangement that spans the eukaryotic kingdom [11]. Three conserved classes of NPC proteins were previously described in yeast and humans [6] : (i) membrane-bound nucleoporins, which link the nuclear pore to the NE; (ii) core-scaffold NUPs, which are restricted at the structural level; and (iii) NUPs that are distributed on the cytoplasmic or nucleoplasmic face of the NE. The three distinct structural arrangements found in eukaryotic core-scaffold NUPs ( $\beta$-propeller fold, $\alpha$-solenoid fold and a mixture of both) were found to be conserved in T. brucei. Moreover, the sequence of some T. brucei core-scaffold NUPs also encompasses phenylalanine-glycine (FG) repeats, which is typical of NUPs [11]. The FG NUPs are known to regulate the

101 transport of molecule through the nuclear pore by extending their FG repeats region into the channel [14] . These 102 results point to a possible ancient inheritance of the structural components of the NPCs [11]. However, it is still 103 unknown whether this apparent structural conservation between Opisthokonts (humans and yeast) and 
104 Discicristates (Trypanosoma) also holds true for other distant eukaryotes. Apicomplexan parasites (Alveolates)

105 may provide another point of comparison between eukaryotic groups. However, although the dynamics of the

106 nuclear pores were described during the cell cycle of the erythrocytic forms of P. falciparum [15] and for an

107 individual component of the nuclear pore (PfSec13) [16], the composition of the P. falciparum NPC remains

108 unknown. In T. gondii, the NPC has not been studied, and its components remain to be identified.

109 We characterized the NPC in T. gondii by identifying the protein complex associated with a conserved component 110 of the core-scaffold NUPs. In this study, we show that TgNup302 is an essential protein that presents a typical 111 perinuclear staining. In a conditional TgNup302 mutant, controlled bidirectional nuclear-cytoplasmic transport 112 was severely impaired, and gene expression was also perturbed. Immunoprecipitation of proteins associated with 113 TgNup302 provide further characterization of the NPC in T. gondii and indicate that structural conservation among 114 eukaryotic NUPs is also true for Alveolates.

115

\section{$116 \quad$ Materials and Methods}

Toxoplasma gondii strain RHAKu80 TaTi (a strain with ATc inducible system and high homologous 121 recombination; [17]) tachyzoites were propagated in vitro in human foreskin fibroblasts (HFF) using Dulbeccos's modified Eagles medium supplemented with 10\% fetal calf serum (FCS), 2mM glutamine, and 1\% penicillinstreptomycin. T. gondii tachyzoites were grown in ventilated tissue culture flasks at $37^{\circ} \mathrm{C}$ and $5 \% \mathrm{CO}_{2}$. Transgenes

124 were introduced by electroporation into tachyzoites of $T$. gondii strains and stable transformants were selected by culture in the presence of $2 \mu \mathrm{M}$ pyrimethamine or chloramphenicol $(34 \mathrm{mg} / \mathrm{ml})$. Clonal lines were obtained by

126 limiting dilution. Prior to RNA and protein purification, intracellular parasites were purified by sequential syringe passage with 17-gauge and 26-gauge needles and filtration through a 3- $\mu \mathrm{m}$ polycarbonate membrane filter.

Generation of transgenic T. gondii strains

131 The TgNup302 iKD line was generated using RH $\Delta$ ku80TaTi and a plasmid containing genomic fragments 132 encompassing $2 \mathrm{~kb}$ upstream the gene and $2 \mathrm{~kb}$ from the predicted ATG [17]. To produced Myc-tagged TgNup115, 133 TgNup134, TgNup129, TgFACT140, TgNup503, TgNup407, TGGT1_311625 proteins by the knock-in strategy, 
134 a DNA fragment of $2 \mathrm{~kb}$ upstream of the stop codon from the genomic sequence was amplified from genomic DNA of $\Delta \mathrm{ku} 80 \mathrm{RH} T$. gondii (type I strain) and cloned in the pLIC-Myc-CAT plasmid. The plasmid $(25 \mu \mathrm{g})$ was

136 linearized with NheI, ApaI, NcoI, BstBI, NcoI, NsiI, NarI, respectively, and transfected in $5.10^{6}$ TgNup302 iKD tachyzoites followed by chloramphenicol selection. The sequences of all primers used in this study are listed in Supplementary Table 1.

139

142 Plaque assays were performed using 6-well plates containing human fibroblast cells infected with 200 parasites

143 per well in media with or without $1 \mu \mathrm{g} / \mathrm{ml}$ ATc, fixed after 7 days post infection and labelled with a crystal violet 144 solution.

145 For parasite growth assays, $8.10^{5}$ parasites per well in a 24 -well plate were incubated $4 \mathrm{~h}$ in normal media or media 146 with ATc. After coverslips were incubated for 24,48 or $72 \mathrm{~h}$, fixation and staining were carried out using 147 formaldehyde and an antibody directed against the TgENO2. The number of parasites per vacuole was counted for 148100 vacuoles per condition.

\section{Antibodies}

\section{1}

152 The anti-TgENO2 rabbit [18], anti-TgChromo1 mouse [19] and anti-TgNF3 [20] mouse antibodies were used at 153 1:1000, 1:200 respectively. Anti-HA rabbit (Eurogentech) and rat (Invitrogen) antibodies were used at 1:500 in 154 IFA and in Western blots. Anti-Myc mouse (ThermoFisher) was used at 1:200 in IFA and 1:500 in Western blot.

158 Intracellular and extracellular parasites tachyzoites were fixed with $4 \%$ paraformaldehyde in PBS for 15 min, 159 followed by two PBS washes. Extracellular parasites were dried on Teflon slides. Parasites were permeabilized 160 with $0.1 \%$ Triton X-100 in PBS containing $0.1 \%$ glycine for $10 \mathrm{~min}$ at room temperature. Samples were blocked 161 with FCS in the same buffer and the primary antibodies were added on parasites in the same buffer for $1 \mathrm{~h}$ at room 162 temperature. Secondary antibody coupled to Alexa-488 or to Alexa-594 (Molecular probes) diluted at 1:1000 was 163 added in addition to DAPI for nucleus staining. Confocal imaging was performed with an LSM880 microscope 
164 (Zeiss) and a Plan Apochromat objective (Plan-Apochromat 63x:1.40 Oil DIC M27, Zeiss). Ultrastructural morphology was performed using conventional microscopy [20], except that 8\% paraformaldehyde containing $0.01 \%$ glutaraldehyde was used for cryo-IEM.

167

170 SIM was used to obtain high-resolution images using an ElyraPS1 microscope system (Zeiss) with a 100x oil171 immersion lens (alpha Plan Apochromat 100x, NA 1.46, oil immersion) and a resolution of $120 \mathrm{~nm}$ along the $\mathrm{x}-\mathrm{y}$ 172 axis and $500 \mathrm{~nm}$ along the z-axis (PSF measured on $100 \mathrm{~nm}$ beads; Sampling voxel size: 0,050 $\mu \mathrm{m} * 0,050$ $173 \mu \mathrm{m} * 0,150 \mu \mathrm{m})$. Three lasers $(405,488$, and $561 \mathrm{~nm})$ were used for excitation. SIM images were acquired with an 174 EMCCD camera (Andor Technology Ltd, UK) and processed with ZEN software; exposure times varied between 175100 and $120 \mathrm{~ms}$. Three-dimensional images were generated using a z-step of $150 \mathrm{~nm}$ (total thickness $\sim 5 \mu \mathrm{m}$ ), 176 while reconstructions and co-distributions were determined with IMARIS software (Pearson's coefficient). We 177 determined the same A (red-HA signal) and B (green-Myc signal) thresholds for all parasite strains. The acquisition 178 was performed sequentially using Zeiss Filter Sets 43HE, 38HE and BP 420-480. Fifteen frames were acquired to 179 reconstruct one image (5 rotations $\mathrm{x} 3$ phases, with a SIM grating period of $51 \mu \mathrm{m}$ for the blue channel, $42 \mu \mathrm{m}$ for 180 the green channel, $34 \mu \mathrm{m}$ for the red channel). One hundred nanometer beads were imaged to measure the 181 chromatic misalignment of our system (fit procedure by the Zen software); these parameters enabled us to further 182 correct the alignment on each acquired multi-channel stack.

186 Intracellular parasites were treated with or without ATc for 48 hours and purified as described earlier. The parasites 187 were fixed with $4 \%$ paraformaldehyde for $30 \mathrm{~min}$ and settled on RNA-treated slides as previously described [21]. 188 Cy3-oligo d(T)40 and Cy3-18S RNA were hybridized on the slide at $50^{\circ} \mathrm{C}$ overnight and washed as previously 189 described [22][21]. After 10 min DAPI staining, the slides were mounted on coverslips. 
193 Intracellular parasites ( $5 \times 10^{8}$ tachyzoites) of the TgNup302-HA $\Delta$ Ku80RHTaTi strain were purified on a 3-mm

194 filter and washed twice with PBS. The parasite pellet was resuspended in $1 \mathrm{~mL}$ of NEB1 buffer (10 mM HEPES $195 \mathrm{pH7.9,} 1.5 \mathrm{mM} \mathrm{MgCl} 2,10 \mathrm{mM} \mathrm{KCl}, 0.5 \mathrm{mM}$ DTT, $0.1 \mathrm{mM}$ EDTA, 0.65\% NP40 and $0.5 \mathrm{mM}$ PMSF), incubated 196 on ice for $10 \mathrm{~min}$ and centrifuged at $1500 \mathrm{~g}$ for $10 \mathrm{~min}$ at $4{ }^{\circ} \mathrm{C}$. The supernatant was kept as the cytoplasmic extract.

197 The pellet was then resuspended with $100 \mu \mathrm{l}$ of buffer NEB2 (20 mM HEPES pH7.9, $1.5 \mathrm{mM} \mathrm{MgCl}, 420 \mathrm{mM}$

$198 \mathrm{NaCl}, 0.2 \mathrm{mM}$ EDTA, $0.5 \mathrm{mM}$ DTT, 25\% glycerol and $0.2 \mathrm{mM}$ PMSF) for $10 \mathrm{~min}$ on ice and centrifuged at 12000 $199 \mathrm{~g}$ for $10 \mathrm{~min}$ at $4^{\circ} \mathrm{C}$.

200 The supernatant was kept as the nuclear extract. The insoluble material was extracted using an SDS buffer (2\% 201 SDS, $10 \mathrm{mM}$ Tris and $0.2 \mathrm{mM}$ PMSF) for $20 \mathrm{~min}$ at room temperature and centrifuged at $12000 \mathrm{~g}$ for $10 \mathrm{~min}$. and 202 the supernatant was kept. Then, $30 \mu 1$ of Pierce anti-c-Myc tag beads were washed twice with 1X TBS (50 mM 203 Tris- $\mathrm{HCl}, 150 \mathrm{mM} \mathrm{NaCl}$ and $0.5 \mathrm{mM}$ PMSF) with centrifugation of $4000 \mathrm{~g}$ for $1 \mathrm{~min}$ between each wash. 204 Saturation of the beads was established with $500 \mu \mathrm{L}$ of $1 \mathrm{X}$ TBS and $5 \mu \mathrm{L}$ of BSA ( $1 \mathrm{mg} / \mathrm{mL})$ under stirring for 15 $205 \min$ at $4{ }^{\circ} \mathrm{C}$, and then the beads were washed twice as previously. Nuclear extract and insoluble material are added 206 to the anti-c-Myc beads (the volume was extended to approximately $900 \mu \mathrm{l}$ of $1 \mathrm{X}$ TBS to dilute the salt 207 concentration) and incubated overnight at $4{ }^{\circ} \mathrm{C}$ under stirring. The next day, the beads were washed five times with 208 1X TBS-T (1X TBS, Tween 20\% and $0.5 \mathrm{mM}$ PMSF) and one time with $62.5 \mathrm{mM}$ Tris pH $6.8+$ PMSF, with 209 centrifugation at $4000 \mathrm{~g}$ for $1 \mathrm{~min}$ between each wash. Finally, the immunoprecipitated proteins from the beads 210 were eluted with $1 \mathrm{X}$ DTT (Tris $0.5 \mathrm{M} \mathrm{pH} 6.8$, SDS $20 \%$, saccharose, DTT $1 \mathrm{M}$ ), warmed at $95^{\circ} \mathrm{C}$ for 5 min and 211 centrifuged at $14000 \mathrm{rpm}$ for $1 \mathrm{~min}$. The supernatants were kept to perform a Western blot.

213 gRNA CRISPR/Cas9 screening

215 To generate guide RNAs (gRNAs) to disrupt specific TgNup115, TgNup302, TgNup593, TgSec13, TgNup530, 216 TgNup37, TgNup68, TgNup216, TgNup67, TgFACT140, TGGT1_228100, TgNup129, TgNup134, TgNup407, 217 TgNup503 and TGGT1_311625 genes, we modified the CRISPR Cas9-gRNA plasmid (pSAG1::Cas9218 U6::sgUPRT plasmid) as previously described [23]. To study the impact of targeted mutations at a specific locus, 219 RH $\Delta$ hxgprt parasites $\left(10.10^{6}\right)$ were transfected with $100 \mu \mathrm{g}$ of CRISPR plasmids generated previously by 220 electroporation. To estimate the frequency of CRISPR/CAS9-mediated gene disruptions, $30 \mu \mathrm{L}$ of transfection 221 reagent was added to HFF monolayers and analyzed by immunofluorescence staining for GFP $24 \mathrm{~h}$ post222 transfection. 
Mass spectrometry proteomic analysis

After denaturation at $100^{\circ} \mathrm{C}$ in $5 \%$ SDS, $5 \% \beta$-mercaptoethanol, $1 \mathrm{mM}$ EDTA, $10 \%$ glycerol, $10 \mathrm{mM}$ Tris pH 8 227 buffer for $3 \mathrm{~min}$, protein samples were fractionated on a 10\% acrylamide SDS-PAGE gel. The electrophoretic migration was stopped as soon as the protein sample entered $1 \mathrm{~cm}$ into the separating gel. The gel was briefly labeled with Coomassie Blue, and five bands, containing the whole sample, was cut. In gel digestion of gel slices was performed as previously described [24].

An UltiMate 3000 RSLCnano System (Thermo Fisher Scientific) was used for separation of the protein digests. Peptides were automatically fractionated onto a commercial C18 reversed phase column $(75 \mu \mathrm{m} \times 150 \mathrm{~mm}, 2 \mu \mathrm{m}$ particle, PepMap100 RSLC column, Thermo Fisher Scientific, temperature $35^{\circ} \mathrm{C}$ ). Trapping was performed during 4 min at $5 \mu \mathrm{L} / \mathrm{min}$, with solvent $\mathrm{A}(98 \% \mathrm{H} 2 \mathrm{O}, 2 \% \mathrm{ACN}$ and $0.1 \% \mathrm{FA})$. Elution was performed using two solvents $\mathrm{A}(0,1 \% \mathrm{FA}$ in water $)$ and $\mathrm{B}(0,1 \% \mathrm{FA}$ in $\mathrm{ACN})$ at a flow rate of $300 \mathrm{~nL} / \mathrm{min}$. Gradient separation was 3 min at $5 \%$ B, 37 min from $5 \%$ B to $30 \%$ B, 5 min to $80 \%$ B, and maintained for 5 min. The column was equilibrated for 10 min with $5 \%$ buffer B prior to the next sample analysis.

The eluted peptides from the C18 column were analyzed by Q-Exactive instruments (Thermo Fisher Scientific). The electrospray voltage was $1.9 \mathrm{kV}$, and the capillary temperature was $275^{\circ} \mathrm{C}$. Full MS scans were acquired in the Orbitrap mass analyzer over $\mathrm{m} / \mathrm{z}$ 300-1200 range with resolution 35,000 (m/z 200). The target value was $5.00 \mathrm{E}+05$. Ten most intense peaks with charge state between 2 and 4 were fragmented in the HCD collision cell with normalized collision energy of $27 \%$, and tandem mass spectrum was acquired in the Orbitrap mass analyzer with resolution 17,500 at $\mathrm{m} / \mathrm{z} 200$. The target value was $1.00 \mathrm{E}+05$. The ion selection threshold was $5.0 \mathrm{E}+04$ counts, and the maximum allowed ion accumulation times were $250 \mathrm{~ms}$ for full MS scans and $100 \mathrm{~ms}$ for tandem mass spectrum. Dynamic exclusion was set to $30 \mathrm{~s}$.

Proteomic data analysis

Raw data collected during nanoLC-MS/MS analyses were processed and converted into *.mgf peak list format with Proteome Discoverer 1.4 (Thermo Fisher Scientific). MS/MS data was interpreted using search engine Mascot 
on mass measurement of 0.2 Da for precursor and 0.2 Da for fragment ions, against a composite target decoy database (50620 total entries) built with 3 strains of Toxoplasma gondii ToxoDB.org database (strains ME49, GT1 and VEG, release 12.0, September 2014, 25264 entries) fused with the sequences of recombinant trypsin and a list of classical contaminants (46 entries). Cysteine carbamidomethylation, methionine oxidation, protein N-terminal acetylation and cysteine propionamidation were searched as variable modifications. Up to one trypsin missed cleavage were allowed. For each sample, peptides were filtered out according to the cutoff set for proteins hits with 2 or more peptides taller than 7 residues, ion score $>25$, identity score $>0$ and no false positive identification.

\section{Library preparation and RNA Seq}

261 RNA was extracted using Invitrogen Trizol Reagent (cat\#15596018), followed by genomic DNA removal and 262 cleaning using an RNase-free DNase. An Agilent 2100 Bioanalyzer was used to assess the integrity of the RNA samples. Only RNA samples having an RNA integrity score between 8 and 10 were used. Library preparation was

264 performed using the TruSeq Stranded mRNA Sample Preparation kit (Illumina) according to the manufacturer's instructions. Libraries were validated using a Fragment Analyzer and quantified by qPCR (ROCHE LightCycler 480). Clusters were generated on a flow-cell within a cBot using the Cluster Generation Kit (Illumina), and libraries were sequenced as 50-bp reads on a HiSeq 2000 using a Sequence By Synthesis (SBS) technique (Illumina).

268 Image analysis and base calling were performed using the HiSeq Control Software and Real-Time Analysis component. Demultiplexing was performed using Illumina's conversion software (bcl2fastq 2.17). The quality of

270 the data were assessed using FastQC from the Babraham Institute and the Illumina software Sequence Analysis 271 Viewer (SAV). Potential contaminants were investigated with the FastQ Screen software from the Babraham 272 Institute.

273 Contamination by Escherichia coli sequences was evident in the sequenced samples. This contamination, due to a 274 reagent from Life Technologies (SuperScript II enzyme), has been eliminated by aligning sequences (using BWA v0.7.12-r1039 [25]) to the E. coli genome and keeping the unmapped reads for downstream analysis.

RNA-seq reads were aligned to the Toxoplasma gondii genome (ToxoDB-25_TgondiiGT1_Genome.fasta from 277 the ToxoDB Toxoplasma Genomics Resource, downloaded on 08/31/15) with a set of gene model annotations 278 (ToxoDB-25_TgondiiGT1.gff from the ToxoDB Toxoplasma Genomics Resource, downloaded on 08/31/15) 279 using the splice junction mapper TopHat 2.0.13 [26] (with bowtie 2.2.3 [27]). Final read alignments having more 280 than 3 mismatches were discarded. Gene counting was performed using HTSeq-count 0.6.1p1 (union mode) [28]. 
281 Because the data come from a strand-specific assay, the read must be mapped to the opposite strand of the gene.

282 Before statistical analysis, genes with less than 15 reads (combining all the analyzed samples) were filtered out.

283 edgeR differentially expressed genes were identified using the Bioconductor [29] package edgeR [30] 3.6.7. The

284 data were normalized using the Relative Log Expression (RLE) [31] normalization factors. Genes with adjusted 285 p-values less than 5\% (according to the FDR method from Benjamini-Hochberg) were declared differentially 286 expressed.

All primers were designed online using Primer2 v.0.4.0 and are listed in Table S1; the cDNA samples were synthesized from total RNA samples using the RevertAid First Strand cDNA Synthesis Kit (Fermentas). qRTPCR was carried out on an Mx3000P system (Agilent Technologies). Individual reactions were prepared with 0.5 $\mu \mathrm{M}$ of each primer, $\sim 5 \mathrm{ng}$ of cDNA and SYBR Green PCR Master Mix (Applied Biosystems, CA) to a final volume of $20 \mu \mathrm{l}$. All experiments were performed twice with separate biological replicates. For each experiment, reactions were performed in triplicate, and the expression of individual genes was normalized to the housekeeping

\section{Quantifications}

The differences of localization for TgNup115, TgNup134, TgNup407 and TgNup503 proteins with or without ATc treatment were calculated with Image J Software. We designed a Macro which after manually determining the parasite edge (polygon tool and manual drawing on the phase contrast image) and the nucleus edge (threshold based on DAPI signal) is able to determine the quantity of Myc-tagged signal (in green) in the nucleus and the cytoplasm of parasites for each strains.

303 For each parasite we measured the area in $\mu \mathrm{m}^{2}$ of regions of interest (nucleus and cytoplasm) and the integrated

304 and mean intensities in gray levels in each region. With the different values, we calculated the ratio cytoplasm

305 signal versus the nuclear signal with or without ATc treatment for each strains. This ratio corresponds to the 306 integrated intensity for the cytoplasm vs integrated Intensity for the nucleus.

\section{Statistics}

309 A non-parametric Student's T-test was performed where statistical analysis was required. 
$\underline{\text { Results }}$

315 Computational searching through the Toxoplasma genome database (toxodb.org) using the human Nup98/96

316 protein as a template revealed the existence of a protein (TGGT1_259640) that is conserved in most eukaryotes

317 from yeast to mammals as an integral component of the NPCs. We constructed a phylogenetic tree including some

318 members of the Apicomplexa, Fungi, Plantae and Eumetazoa, where there is a level of characterization of the NPC

319 proteome and some direct experimental information [10] [8] [32] [33] [11]. The Nup98/96 homologs from the

320 Apicomplexa taxon, joining representative branch species such as Toxoplasma, Neospora, Eimeria, Theileria and

321 Babesia, are relatively close to Eumetazoa, followed by Plantae. Surprisingly, members of the Plasmodium

322 species, which belongs to the Apicomplexa phylum as Toxoplasma, present a distant branching taxon separated

323 from the Apicomplexa (Fig. S1). The TgNup302 gene (TGGT1_259640) is predicted to produce a long 2894-

324 amino-acid protein containing an autocleavage domain (Pfam domain: PF04096), that may produce two distinct

325 N-terminal (1079 aa) and C-terminal (1620 aa) peptides after self-cleavage. The presence of GLFG repeats, a

326 typical variant of the FG repeats present in the human Nup98 protein, at its N-terminus and a Nup96-like domain

327 (PF12110) identified by the Pfam database at its C-terminus is typical of this protein family. This structure is

328 conserved among eukaryotes, with Nup98/96 and NUP145 being the representative proteins in humans and yeast,

329 respectively. In vertebrates, Nup98 binds directly to Nup96, the C-terminal half of the proteolytically processed

330 Nup98/Nup96 polyprotein, and is a component of the Nup107-160 complex (the Nup84 complex in yeast) [34]

331 [9]. However, the size of the unprocessed $T$. gondii protein (302 kDa) is much larger than in yeast (145 kDa) and

332 in humans $(194 \mathrm{kDa})$.

333 To better characterize the biological role of this protein, we used a promoter replacement strategy to produce a

334 conditional knock-down (iKD) mutant strain. In this strain, the expression of the TgNup302 transcript is under the

335 control of anhydrotetracycline (ATc) (Fig.S2) [17]. When ATc is added to the culture media, the regulatable

336 promoter is repressed, and the TgNup302 transcript is no longer produced. We simultaneously added an HA-tag

337 to the N-terminus of the protein in order to follow the protein expression (Fig. S2). Because the human and yeast

338 homologous proteins undergo a self-proteolytic cleavage, producing two polypeptides, we also tagged the

339 TgNup302 protein with a Myc tag at its C-terminus (Fig. S2) in the iKD strain. Therefore, we produced a strain

340 expressing TgNup302 under the control of ATc and tagged at its N- and C-termini. The correct insertion of the 
341 construction at the TgNup302 locus was validated by genomic PCR (Fig.S2). Using anti-HA ( $\alpha$-HA) and anti-Myc

$342(\alpha-\mathrm{Myc})$ monoclonal antibodies by immunofluorescence assay (IFA), we observed that TgNup302 presented a

343 punctate perinuclear pattern in the parasite, consistent with nuclear pore staining (Fig. 1a). Under ATc treatment,

344 a substantial decrease in TgNup302 protein expression in iKD parasites was observed for both HA- and Myc-

345 tagged proteins. Western blots of total protein extracts from this transgenic parasite line revealed the expression 346 of the two polypeptides tagged with HA- or Myc-tags of the predicted size (150 and $170 \mathrm{kDa}$, respectively) (Fig.

347 1b) in the absence of ATc indicating the cleavage of the TgNup302 protein into two polypeptides. Notably, 348 TbNup158, the T. brucei homologue of TgNup302, does not perform self-cleavage and remains as one polypeptide

$349[11]$ and therefore is closer to the yeast Nup116 or Nup100 protein.

350 In the presence of ATc, we observed a drastic reduction in the signal from both tagged proteins, with an 351 undetectable level after 48 hours of treatment (Fig. 1b). As a first assessment of the requirement for the $\operatorname{TgNup} 302$ 352 locus, we tested the ability of parasites to grow and produce plaques on host cell monolayers. After 7 days, the 353 wild-type parasites grew normally and developed equal sized plaques in the absence or presence of ATc. Similarly, 354 the growth of the iKD line gave rise to normal plaque numbers only in the absence of ATc. In the presence of ATc, 355 the TgNup302-deficient parasites were unable to proliferate and form plaques (Fig. 1c), indicating a drastic 356 impairment of growth and/or invasion of the parasites lacking the expression of TgNup302. This result was 357 confirmed by an experiment recording the number of parasites per vacuole at a given time (Fig. 1d). While the 358 parental strain with or without ATc showed similar growth to that of the iKD strain without ATc for 48 hours, we 359 noticed that the iKD-HA strain produced vacuoles with a smaller number of parasites. This indicates that the 360 growth of this parasite is impaired by the absence of TgNup302 (Fig. 1d).

TgNup302 is involved in nuclear import and essential to $18 S$ RNA export

364 To validate the role of TgNup302 in the nuclear import and export of macromolecular complexes, we examined 365 the localization of a nuclear marker in the iKD strain. Previous work has established the predominant nuclear 366 location of the glycolytic isoenzyme enolase 2 (TgENO2) [35]. We tested the localization of the TgENO2 protein 367 by IFA using a specific antibody ( $\alpha$-TgENO2) in the presence and absence of ATc for 48 hours. We observed 368 predominant nuclear localization of the protein in the parental strain and in the iKD strain in the absence of ATc, 369 as expected (Fig. 2a). However, parasites of the iKD strain grown in the presence of ATc showed a marked 370 delocalization of TgENO2 into the cytoplasm of the parasite (Fig. 2a). This result was confirmed after 
371 quantification of the parasites which showed the nuclear or cytoplasmic localization of TgENO2 (Fig. 2b). While

372 the iKD strain without ATc and the parental parasites exhibited predominant nuclear localization of TgENO2,

373 most of the ATc-treated iKD parasites exhibited cytoplasmic localization, indicating a defect in the transport of 374 this protein in the mutant parasites.

375 To investigate whether TgNup302 is involved in mRNA export, we examined the cellular distribution of 376 poly(A)+RNA in the parental and TgNup302 iKD strains via RNA fluorescence in situ hybridization (FISH) with 377 labeled oligodT after 48 hours with or without ATc treatment. In the parental and iKD strains, localization of the 378 polyA+ RNA was mostly cytoplasmic (Fig. S3). We also examined the localization of the 18S ribosomal RNA 379 using a specific probe. In the wild-type and iKD strains without 48 hours of ATc treatment, most 18S RNA was 380 found to be cytoplasmic (Fig. 2c). In contrast, the 18S RNA signal was mainly nuclear in the iKD parasites treated 381 with ATc (Fig. 2c and d). These results demonstrate that TgNup302 is essential to 18S RNA export, a typical role 382 of the NPC. To test whether TgNup302 was involved in other proteins nuclear import, we used IFA of the parental 383 and $\mathrm{KKD}-\mathrm{HA}$ strains to evaluate the localization of a centromeric protein ( $\mathrm{TgChromo1}$ ) that was previously 384 described to specifically bind pericentromeric heterochromatin [19]. We also tested the localization of a nucleolar 385 marker, (TgNF3). No differences in the localization of these proteins was observed between the parental and iKD386 HA strains in all conditions tested (with or without ATc for 48 hours) (Fig. S4).

387 The morphology of the parasite was assessed using electron microscopy for parental and TgNup302 iKD 388 intracellular parasites following $48 \mathrm{~h}$ of growth with or without ATc treatment. No morphological differences were 389 observed between the parental and TgNup302 iKD strains, which present a typical nucleus. ATc treatment had 390 also no particular impact on the presence of other organelles, such as rhoptries and micronemes, for each strain, 391 suggesting that TgNup302 is not essential for nuclear morphology (Fig. S5).

394 RNA sequencing (RNA Seq) was carried out to study gene expression dynamics in the TgNup302 iKD. Total 395 RNA was purified from tachyzoites of the TgNup302 iKD strain grown under normal growth conditions with or 396 without ATc treatment for $48 \mathrm{hr}$ (in triplicate). RNA-seq reads were aligned to the Toxoplasma gondii genome 397 with a set of gene model annotations using the splice junction mapper TopHat 2.0.13. The data were normalized using the relative log expression (RLE) normalization factors. Genes with adjusted p-values less than 5\% 399 (according to the FDR method from Benjamini-Hochberg) were considered differentially expressed. Data analysis revealed significant changes in the transcription profile of $T$. gondii, with 145 genes upregulated and 65 genes 
downregulated (Table S2). The predominance of upregulated genes suggests a role for the NPC in the repression of gene expression. We examined the differential chromosomal distribution of differentially expressed genes and did not identify a cluster of differentially regulated genes (Fig. S6). Collectively, these data suggest that TgNup302 could play a role as a repressor and its absence modulates the transcriptional regulation of genes that are normally repressed at the tachyzoite stage of $T$. gondii. Validation of the RNA Seq data was also carried out using qRTPCR in RNA samples from the iKD TgNup302 strain with and without ATc treatment for 48 hours. For that, we selected 5 upregulated genes with a $\operatorname{logFC}>-2$ (TGGT1_258670, TGGT1_301150, TGGT1_267160, TGGT1_270273, TGGT1_360460) (Fig. 3a) and TgNup302 (TGGT1_259640) (Figure 3b) as a control. As expected, Figure 3a clearly shows that ATc treatment induced an increased steady-state mRNA level of all five upregulated genes, while the TgNup302 transcript was downregulated in the iKD samples treated with ATc.

New components of the T. gondii nuclear pore

NPC components are unknown in $T$. gondii. To uncover the components of the NPC in $T$. gondii, we performed co-immunoprecipitation (co-IP) experiments on the iKD Nup302-HA parasites using $\alpha$-HA antibody followed by mass spectrometry identification of the co-IP proteins (from two experiments). As a control, we performed an $\alpha$ HA immunoprecipitation on protein extracts from the parental strain, RH $\Delta \mathrm{Ku} 80 \mathrm{TaTi}$. We selected the proteins identified in both experiments but not identified in the control and those present in the control with a single peptide (Table S3 and Table 1). Among the proteins identified and listed in Table 1, we confirmed the presence of TgNup302 (TGGT1_259640) with peptides spanning both the N-terminus and the C-terminus (Fig. S7), indicating that both products of TgNup302 autocleavage are present in the IP fraction. As shown for the human Nup98/Nup96 protein [36], both polypeptides may also interact in T. gondii.

Most of the identified proteins did not share primary sequence conservation with known nucleoporins using BLAST searches. However, a conserved structure was identified between distant eukaryotic NUPs [11], we therefore examined the secondary structure prediction of the selected proteins (Table 1, Fig. S8). We also searched the proteins for other motifs using the Pfam database. Among the co-immunoprecipitated proteins, five putative 427 NUPs encompassing FG-repeat motifs (TgNup593, TgNup37, TgNup68, TgNup216 and TgNup67) were 428 identified. We also found proteins with a $\beta$-propeller fold (TgSec13, TGGT1_311625 and TGGT1_228100), including a homolog of the yeast protein Sec13, shown to be part of the Y complex through its interaction with Nup96 [37]. Interestingly, six of the TgNup302-associated proteins (TgNup530, TgNup407, TgNup129, 
431 TgNup503, TgNup115 and TgNup134) had no recognizable Pfam motif, with the exception of TgNup115, which 432 contains a Zinc-finger domain $(\mathrm{CCCH})$. However, these proteins do contain an $\alpha$-solenoid fold, a structure also 433 found in other eukaryotic core-scaffold NUPs (Fig. S8). Moreover, two of these proteins exhibited large segments 434 of coiled-coil structure (TgNup530, TgNup407) (Fig. S8). We further examined the sequence of these proteins 435 using a secondary structure prediction software [38]. This allowed the identification of a domain in TgNup68 with 436 a strong homology to the yeast Nsp1 crystal structure (Table 1). Similarly, a domain in TgNup129 was homologous 437 to the yeast Nic96 structure (Table 1). These data indicate that the proteins composing the T. gondii NPC may 438 have retained the same level of structural conservation as T. brucei NUPs. Notably, the size of the identified 439 proteins substantially exceeded the size of their putative homologs. Therefore, the assigned homologs listed in 440 Table 1 are based on the structural properties of the proteins rather than their expected size.

441 As expected, TgNup302 is also associated with the putative homolog of the ribonucleic acid export 1 protein 442 (TgRae1, TGGT1_272350), which is implicated in the mRNA export pathway in other eukaryotes, which also 443 interact with the GLFG Nup98 protein [39]. Surprisingly, we also found the two proteins (TgFACT140 and 444 TgFACT80) corresponding to the homologs of the proteins composing the FACT complex, which is categorized 445 as a histone chaperone critical for nucleosome reorganization during replication and transcription [26] [27] [28]. 446 This observation indicates a potential interaction between the FACT complex and the NPC in T. gondii.

Identified proteins interact with TgNup302

450 To confirm the mass-spectrometry results, we performed a reverse IP of TgNup302 using the identified partner 451 proteins. Protein extracts from parasite strains expressing each Myc-tagged protein in the TgNup302-HA iKD 452 background were immunoprecipitated using anti-Myc tag beads. The TgNup302-HA iKD strain was used as a 453 negative control. We then performed a Western blot using an anti-HA antibody to detect TgNup302 in the eluates 454 of the Myc-tagged immunoprecipitates (Fig. 4). As shown in Figure 4a, the anti-Myc antibody failed to 455 immunoprecipitate the HA-tagged TgNup302 in the absence of Myc-tagged proteins, as expected. In contrast, we were able to confirm the interaction between TgNup302 and the TgFACT140 protein, indicating a link between 457 the NPC and the FACT complex in T. gondii (Fig. 4a, line 2, top panel). We tested whether some of the unknown proteins with an $\alpha$-solenoid fold listed in Table 1 were also able to co-immunoprecipitate TgNup302. We 459 confirmed the presence of the TgNup302 protein in the TgNup129 co-immunoprecipitated proteins (Fig. 4a, line 460 3). Similarly, TgNup302 was detected after immunoprecipitation of TgNup134 and TgNup407, indicating that 461 these proteins of unknown function may participate in the NPC (Fig. 4a, lines 4 and 5, top panel). Western blot 
membranes were probed with an anti-Myc antibody to detect specific Myc-tagged proteins (Fig. 4a, bottom panel).

463 Using the anti-Myc antibody, the immunoprecipitated proteins extracted from the TgNup302 iKD parasite line 464 showed no signal, as expected (Fig. 4a, line 1, bottom panel). We confirmed the presence of each Myc-tagged protein at the expected molecular weights for TgFACT140 (135 kDa), for TgNup129 and TgNup134 (130 kDa) and for TgNup407 (115 kDa). We verified that the level of the TgNup302 protein was similar in the starting material of each strain (Figure S9a). Similarly, the presence of the myc-tagged proteins was checked for the same input samples (Figure S9b). As a control, we repeated this experiment using a strain expressing TgNup302-HA and a myc-tagged protein (TgChromo1) that was not identified by mass-spectrometry (Figure 4b). After immunoprecipitation with anti-myc coated beads, we performed a Western blot using an anti-HA antibody to detect TgNup302 in the input and eluates (Fig. 4b left panel). We were able to confirm the interaction between TgNup302 and the TgNup134 protein (Fig. 4b, line 2, left panel). As expected, TgNup302 is not detected in the negative control as well as in the TgChromo1 co-immunoprecipitated proteins (Fig. 4b, lines 4 and 6 left panel)

474 but is present in the input (Fig. 4b, lines 1, 2, 3, left panel). Western blot membranes were probed with an anti475 Myc antibody to detect specific Myc-tagged proteins in the input and eluates (Fig. 4b, right panel). Using the anti476 Myc antibody, the immunoprecipitated proteins extracted from the TgNup302 iKD parasite line showed no signal, as expected (Fig. 4b, line 1 and 4, right panel). We confirmed the presence of each Myc-tagged protein in the 478 eluates for TgChromo1 (Fig. 4b, line 6, right panel) and TgNup134 (Fig. 4b, line 5, right panel) confirming that 479 the myc-tagged proteins were indeed immunoprecipitated.

480 These results confirm the interaction between the NPC protein TgNup302 and the selected binding partners 481 identified by mass-spectrometry.

Identified proteins localize to the T. gondii nuclear pore

485 To gain more information about these unknown proteins presenting an $\alpha$-solenoid fold, we tagged several of them 486 using a Myc-tag in the iKD TgNup302-HA strain. As a control, we tagged a component of the FACT complex 487 (TgFACT140) whose localization was anticipated to be nuclear. Using IFA, we observed that the five potential 488 partners exhibited a TgNup302-like pattern (Figs. 5a, 6a and S9) in the absence of ATc (after 48 hours of growth), 489 indicating that they may indeed be components of the NPC. Therefore, we propose to rename them as TgNup 490 proteins, as suggested in Table 1. As anticipated, the TgFACT140 protein showed strong nuclear localization that 
491 extended to the perinuclear periphery (Fig. S9). Notably, the TgNup503 protein showed a more spread pattern that extended toward the cytoplasm of the cell (Fig. 5a) and might not be connected to the nuclear envelope.

493 We next examined whether the localization of these proteins was perturbed in the TgNup302-deficient parasites.

494 We performed IFA and measured the quantity of signal in the cytoplasm and in the nucleus (as defined by DAPI 495 staining) in the absence and presence of ATc (Fig. 5b) in multiple vacuoles. We identified two proteins 496 (TgNup115, TgNup134) with a greater proportion of the fluorescence in the cytosol in the presence of ATc (Fig. 497 5b). In contrast, TgNup503 presented a distribution that remained unchanged after ATc treatment (Fig. 5b). 498 Notably, we observed that TgNup407 localization was perinuclear in the absence of ATc and changed in the 499 presence of ATc, with a more pronounced localization inside the nucleus (Fig. 6a). We measured the quantity of 500 signal present at the nuclear periphery and inside the nucleus in the presence and absence of ATc (after 48 hours) 501 (Fig. 6b). We found that there was much more signal in the nucleus than at the nuclear periphery in the presence 502 of ATc compared to in the absence of ATc (Fig. 6b). We examined the same ratio for TgNup129 protein 503 localization, which exhibited a punctate perinuclear pattern that remained unchanged by ATc treatment (Fig. S10).

504 Similarly, TgFACT140 exhibited a nuclear localization that was not perturbed by ATc treatment (Fig. S10).

505

506

507

508

509

510

\section{Structured illuminated microscopy imaging of TgNup302 and its binding partners.}

To better characterize the structure of the NPC in $T$. gondii, we performed structured illuminated microscopy (SIM) on selected parasite strains. For each strain, colocalization between TgNup302-HA and its Myc-tagged protein partner was determined by Imaris software (Pearson's coefficient). Hence, we determined a Pearson's coefficient average of 0.65 for the Myc-tagged TgNup302 C-terminus, 0.46 for TgNup67, 0.63 for TgNup129, 0.28 for TgNup503, 0.23 for TgNup1 15 and 0.56 for TgNup134 with the HA-tagged TgNup302 protein (Fig. 7a). Using SIM microscopy, we observed for four proteins (TgNup302 C-terminus, TgNup67, TgNup129 and TgNup134) between 4 and 8 perinuclear co-distribution foci (indicated with white arrows) per parasite, representing co-distribution between HA (red) and Myc (green) staining (Fig. 7b). This may indicate that the parasite has 4 or 8 complete NPC structures per nuclei, a number that is comparable to what was observed in $P$. falciparum (Fig. 7c) [15] [40]. In contrast, TgNup115 and TgNup503 had lower Pearson's coefficients, displaying a lower number of foci per parasite and indicating that these proteins may only be transiently interacting with NPCs (Figs. 7b and c). 
522 shuttling.

524 Previous studies have shown that combining CRISPR/CAS9 with sgRNAs can be used to generate site-specific 525 double-strand breaks in the target DNA that are repaired by non-homologous-end joining, leading to short 526 insertions and deletions that inactivate the gene [41]. Recently, the CRISPR/CAS9 system was adapted to T. gondii 527 with a plasmid expressing a nuclear-localized CAS9 fused to green fluorescent protein (CAS9-NLS-GFP) driven 528 by the SAG1 promoter and a single guide RNA (sgRNA) driven by the T. gondii U6 (TgU6) promoter [23]. We 529 used CRISPR/Cas9 and a single gRNA placed downstream of the ATG to target the fifteen proteins previously 530 identified by co-immunoprecipitation with the TgNup302 protein (Table 1). As a control, we used a sgRNA 531 targeting a non-essential gene (TgAlba1) [21]. For each construct, GFP expression was monitored to determine 532 the transfection efficiency at $24 \mathrm{~h}$ after electroporation, revealing that $\sim 30$ to $70 \%$ of cells received the plasmid 533 (Fig. S11). We used IFA to determine the localization of the TgENO2 protein, a nuclear marker. We determined 534 the percentage of vacuoles that were GFP-negative and had cytoplasmic TgENO2 staining, as a control. We also 535 scored the percentage of vacuoles that were GFP-positive (CAS9-expressing) and had cytoplasmic TgENO2 536 staining, illustrating the impact of the potentially mutated gene on the TgENO2 localization (Fig. 8). As expected, 537 no differences were observed between these two scores for the parasite expressing CAS9 and the sgRNA targeting 538 TgAlba1. Similarly, most of the targeted genes did not result in an increase in the number of vacuoles that had 539 cytoplasmic TgENO2 and were GFP-positive. In contrast, the parasite expressing Cas9 and an sgRNA directed 540 against the TgNup302 gene presented a drastic increase of the cytoplasmic TgENO2 signal (Fig. 8), in good 541 concordance with the phenotype observed with the iKD strain (Fig. 2b). Interestingly, for sgRNA targeting the 542 TgNup503 and TgNup134 genes, we observed a higher percentage (93 and 57\%, respectively) of GFP-positive 543 vacuoles with cytoplasmic TgENO2 staining than that of GFP-negative vacuoles with cytoplasmic TgENO2 544 staining (14 and 16\%, respectively) (Fig. 8). Collectively, these results may indicate that in addition to TgNup302, 545 the TgNup503 and TgNup134 proteins are essential for the nuclear localization of TgENO2.

\section{$\underline{\text { Discussion }}$}

550 Nuclear pore complex components have been widely studied in yeast and humans. More recently, NUP proteins 551 were uncovered in a distant eukaryote, T. brucei $[42,43]$. Strikingly, structural conservation is a key feature of $T$. 
553 investigated the role and the composition of the nuclear pore from another distant eukaryotic branch, the

554 Alveolates. Using a conserved nuclear pore protein (TgNup302) homolog of the NUP98/96 and NUP145 proteins

555 in humans and yeast, respectively, we identified potential interacting proteins. Interestingly, TgNup302 has all the

556 features of its human and yeast homologs, including the family-specific GLFG repeats. T. brucei presents only

557 one GLFG repeat protein that does not contain an autocleavage domain, as observed for the yeast Nup100 protein.

558 This indicates that in distant eukaryotes, the GLFG repeat motif is a key feature that was retained during evolution.

559 Because of the stringency of our protocol and as shown by the characterization of the identified proteins, we

560 believe that a significant number of $T$. gondii NUPs were identified during the course of this study. Among these

561 proteins, we purified proteins encompassing FG (phenylalanine-glycine)-repeat motifs, which are distinctive of

562 NPC components. In fact, it was shown that a proportion of NUPs are composed of FG repeats, which function is

563 to mediate the passage of transport receptors and their cargos with selectively gated transport [7].

564 Moreover, other proteins, bearing no recognizable motifs, presented structural features that are shared with

565 eukaryotic NUPs. We further showed that their localization corresponds to known $T$. gondii NUPs, indicating that

566 they may participate in the parasite NPC. This correlates with earlier studies identifying T. brucei NUPs [42,43].

567 Indeed, structural conservation led to the hypothesis of an ancient inheritance of the core-scaffold NUPs. This

568 hypothesis is confirmed by our study identifying NUPs in Alveolates. We also noted that most of the proteins that

569 co-immunoprecipitated with TgNup302 were very large compared with their eukaryotic counterparts. This is also

570 true for the TgNup302 protein itself. This may indicate that fewer proteins are needed to produce a functional

571 nuclear pore in T. gondii.

572 During the course of this study, we also characterized the biological function of TgNup302, demonstrating its role

573 in the nuclear transport of proteins and rRNA. When TgNup302 was depleted, these vital functions were impaired,

574 and as a consequence, the parasite rapidly dies. It is worth noting that the nuclear pore structure is still present in

575 the TgNup302-depleted parasites because some of the nuclear pore proteins exhibited normal localization

576 (TgNup67 and TgNup134). The remaining nuclear pore structure may also still be sufficient for the active transport

577 of the large ribonucleoprotein complexes, insuring the nuclear export of polyA + mRNAs. This indicates that

578 TgNup302 performs specific roles in the complex, as shown for NUP98/96 and NUP145, the representative human

579 and yeast homologs, respectively. In fact, deletion of the essential Nup145 gene results in a defect not in protein

580 import but in polyA+ RNA export [44] [45]. Moreover, NUP98 plays a specific role in RNA export from the

581 nucleus, and it appears to be an essential component of multiple RNA export pathways [46] [47]. NUP98 functions 
as a docking protein for the cytosol-mediated docking of a model import substrate. The docking function was

583 localized to the N-terminal half of NUP98, which contains all its GLFG repeats [48]. However, we cannot exclude

584 the possibility that an undetectable amount of TgNup302 is still present at the nuclear pore after 48 hours of ATc

585 treatment, therefore allowing the passage of mRNAs but not rRNA. We also noted that the nuclear marker

$586 \mathrm{TgENO} 2$ was mainly cytoplasmic in the TgNup302-depleted parasite. However, the localization of a nucleolar

587 marker, TgNF3 or that of a pericentromeric marker (TgChromo1), was not affected after $48 \mathrm{~h}$. This may be

588 explained by the presence of multiple nuclear import pathways in the parasite, as previously suggested by a

589 genomic identification of potential import factors [49]. While the expected molecular weight of TgNF3 (34 kDa)

590 would allow this protein to diffuse freely in the nucleus through the nuclear pore, TgChromo1 $(98 \mathrm{kDa})$ exceeds

591 the size cut-off (approximately $40 \mathrm{kDa}$ ) for passive diffusion of molecules. Alternatively, this might reflect the

592 differences in the dynamics of nuclear import of these proteins, especially in parasites for which growth is altered

593 by TgNup302 depletion.

594 Using SIM imaging, we identified two proteins (TgNup115 and TgNup503) that associate with TgNup302 to a

595 much lesser extent. These proteins may represent transporters that transiently associate with the NPC and should

596 be investigated further, as this class of proteins is under-represented in Apicomplexa genomes [49]. In particular,

597 TgNup115 presents a CCCH zinc finger that may bind RNA and therefore play a role in RNA transport. Overall,

598 we estimated that the number of nuclear pores in $T$. gondii tachyzoites to be between 4 and 8 , a number that is

599 close to what was observed in P. falciparum [15]. However, epitope-tag availability and the microscopy limitations

600 may have hampered the identification of proteins and therefore may have led to an under-estimation of the pore

601 number.

602 To further investigate the potential components of the T. gondii nuclear pore, we performed a CRISPR/Cas9

603 screen. We used the ability of the T. gondii RH strain to perform non-homologous recombination after Cas9

604 cleavage, leading to mutations in the target gene that may impair its expression. Using TgENO2 as a nuclear

605 marker, we identified two other proteins (TgNup503 and TgNup134) that showed a marked defect in TgENO2

606 localization in addition to TgNup302. Given the TgNup503 localization and its association with TgNup302, we

607 believe that these data reinforce the hypothesis that this protein play a major role in the TgENO2 import and

608 potentially other proteins. TgNup503 localization is not dependent on the presence of TgNup302, and therefore it

609 may associate with other proteins of the NPC.

610 The TgNup134 protein strongly associates with TgNup302 and is delocalized in the absence of TgNup302.

611 Therefore, it may associate with the NPC through its interaction with TgNup302 and may represent a peripheral 
612 NUP. TgNup134 may also have a role in nuclear import. Further studies should be performed to validate this 613 hypothesis.

614 In $T$. gondii, previous studies hypothesized a model of centromere sequestration, in which centromere attachment 615 to the centrocone, a subcompartment of the nucleus, serves as an organizer of chromosome apical region location 616 throughout the parasite cell cycle $[19,50]$. We hypothesized that centromeric heterochromatin could be anchored 617 through protein components of the nuclear membranes via nucleoporins. In the TgNup302 iKD mutant, in the 618 presence of ATc, we showed that a marker of the pericentromeric heterochromatin is still sequestered at all times 619 of the cell cycle, indicating that TgNup302 depletion is not sufficient to uncouple the potential link between nuclear 620 pore and centromeric heterochromatin. However, as previously shown, TgNup302 depletion is not sufficient to 621 fully abrogate the NPC presence at the nuclear membrane. Therefore, other proteins in the remaining NPC may 622 still interact with centromeric heterochromatin. Moreover, TgNup302, a FG nucleoporin, may not have a direct 623 access to chromatin as nuclear basket nucleoporin would [51]. Alternatively, proteins directly interacting with the 624 nuclear membrane may be involved in centromere sequestration at the nuclear periphery.

625 Finally, RNA-seq revealed that most of the transcripts that are impacted in the iKD mutant when TgNup302 is 626 depleted are upregulated. This indicates a potential role of the TgNup302 protein in regulating gene expression. . 627 Further experiments are needed to explore the potential role of the NPC in T. gondii gene expression. Because the 628 parasites lacking TgNup302 are severely affected, a kinetic experiment was not performed and would be useful to 629 identify the genes that are impacted early after the depletion of TgNup302. In higher eukaryotes, an alternative 630 splice variant of the Nup98/96 transcript produces a shortened version of the Nup98/96 polypeptide corresponding 631 to the Nup98 protein with a stop codon inserted after the auto-proteolytic domain. This polypeptide is found in 632 the intranuclear pool and can interact with chromatin [52]. Such splicing variants were not detected by in the RNA633 seq data available on ToxoDB. Interestingly, TgNup129 was found to be a structural homolog of the Nup93 (or 634 Nic96 in yeast) protein. This protein was shown to interact at multiple loci on chromatin [53]. Further experiments 635 should be performed to validate this hypothesis.

636 The presence of the HDAC3 protein in the IP (Table S3), may indicate that T. gondii NPCs are recruiting 637 repression-specific complexes at the nuclear periphery. TgHDAC3 has been implicated in the control of the 638 expression of bradyzoite-specific genes during differentiation [54]. Therefore, the NPC may have a role in 639 maintaining the repression state for those loci. However, these genes were scattered in the genome, which may 640 indicate that some genomic regions may contact the nuclear periphery, resulting in the repression of gene 
expression, a mechanism widely recognized in other eukaryotes [51] that was never investigated in $T$. gondii until

now.

643 We also showed that TgNup302 is able to interact with two members of the FACT complex. Indeed, the FACT complex is involved in the reorganization of the nucleosome structure during crucial processes such as DNA replication and transcription elongation [55]. Proteins involved in transcription elongation have already been associated with the NPC [56], but the specific role of the FACT complex at the nuclear periphery was not investigated. Notably, a genetic interaction was identified in yeast between the Pob3 gene (a homolog of TgFACT80) and the Nup100 gene, coding for a GLFG NUP (homologous to TgNup302) [57]. The FACT complex is also known to be required for proper export of mRNA either by favoring an efficient mRNA splicing [58] or through a direct interaction with mRNA export factors [59]. Therefore, we hypothesize that $T$. gondii, and possibly other apicomplexan parasites, may have evolved a strong link between gene transcription, elongation, splicing and export of mRNA. The FACT complex might therefore facilitate the export of mRNPs through the NPC in T. gondii.

This would allow, as shown for other eukaryotes, the rapid expression of genes in T. gondii. In conclusion, we uncovered novel members of the nuclear pore in $T$. gondii and also identified novel proteins that may have a role in the nuclear-cytoplasmic transport of proteins. $\operatorname{TgNup} 302$ is an essential protein that may be used as a tool to investigate the role of the $T$. gondii nuclear pore in regulating gene expression.

\section{Bibliography}

[1] K. Kim, L.M. Weiss, Toxoplasma gondii: the model apicomplexan, Int J Parasitol. 34 (2004) 423-32.

[2] M.S. Behnke, J.B. Radke, A.T. Smith, W.J. Sullivan, M.W. White, The transcription of bradyzoite genes in Toxoplasma gondii is controlled by autonomous promoter elements, Mol. Microbiol. 68 (2008) 15021518. doi:10.1111/j.1365-2958.2008.06249.x.

[3] M.S. Behnke, J.C. Wootton, M.M. Lehmann, J.B. Radke, O. Lucas, J. Nawas, L.D. Sibley, M.W. White, Coordinated progression through two subtranscriptomes underlies the tachyzoite cycle of Toxoplasma gondii, PLoS ONE. 5 (2010) e12354. doi:10.1371/journal.pone.0012354.

[4] W.J. Sullivan, M.A. Hakimi, Histone mediated gene activation in Toxoplasma gondii, Mol Biochem Parasitol. 148 (2006) 109-16.

[5] M. Gissot, K.A. Kelly, J.W. Ajioka, J.M. Greally, K. Kim, Epigenomic Modifications Predict Active Promoters and Gene Structure in Toxoplasma gondii, PLoS Pathog. 3 (2007) e77.

[6] F. Alber, S. Dokudovskaya, L.M. Veenhoff, W. Zhang, J. Kipper, D. Devos, A. Suprapto, O. KarniSchmidt, R. Williams, B.T. Chait, A. Sali, M.P. Rout, The molecular architecture of the nuclear pore complex, Nature. 450 (2007) 695-701. doi:10.1038/nature06405.

[7] M. Capelson, M.W. Hetzer, The role of nuclear pores in gene regulation, development and disease, EMBO Rep. 10 (2009) 697-705. doi:10.1038/embor.2009.147.

[8] M.P. Rout, J.D. Aitchison, A. Suprapto, K. Hjertaas, Y. Zhao, B.T. Chait, The yeast nuclear pore complex: composition, architecture, and transport mechanism, J. Cell Biol. 148 (2000) 635-651.

[9] S.K. Vasu, D.J. Forbes, Nuclear pores and nuclear assembly, Curr. Opin. Cell Biol. 13 (2001) 363-375.

[10] J.M. Cronshaw, A.N. Krutchinsky, W. Zhang, B.T. Chait, M.J. Matunis, Proteomic analysis of the mammalian nuclear pore complex, J. Cell Biol. 158 (2002) 915-927. doi:10.1083/jcb.200206106.

[11] J.A. DeGrasse, K.N. DuBois, D. Devos, T.N. Siegel, A. Sali, M.C. Field, M.P. Rout, B.T. Chait, Evidence for a shared nuclear pore complex architecture that is conserved from the last common eukaryotic ancestor, Mol. Cell Proteomics. 8 (2009) 2119-2130. doi:10.1074/mcp.M900038-MCP200. 
[12] S.O. Obado, M. Brillantes, K. Uryu, W. Zhang, N.E. Ketaren, B.T. Chait, M.C. Field, M.P. Rout, Interactome Mapping Reveals the Evolutionary History of the Nuclear Pore Complex, PLoS Biol. 14 (2016) e1002365. doi:10.1371/journal.pbio.1002365.

[13] J.A. DeGrasse, B.T. Chait, M.C. Field, M.P. Rout, High-yield isolation and subcellular proteomic characterization of nuclear and subnuclear structures from trypanosomes, Methods Mol. Biol. 463 (2008) 77-92. doi:10.1007/978-1-59745-406-3 6.

[14] R.Y.H. Lim, B. Fahrenkrog, J. Köser, K. Schwarz-Herion, J. Deng, U. Aebi, Nanomechanical Basis of Selective Gating by the Nuclear Pore Complex, Science. 318 (2007) 640-643. doi:10.1126/science.1145980.

[15] A. Weiner, N. Dahan-Pasternak, E. Shimoni, V. Shinder, P. von Huth, M. Elbaum, R. Dzikowski, 3D nuclear architecture reveals coupled cell cycle dynamics of chromatin and nuclear pores in the malaria parasite Plasmodium falciparum, Cell. Microbiol. 13 (2011) 967-977. doi:10.1111/j.14625822.2011.01592.x.

[16] N. Dahan-Pasternak, A. Nasereddin, N. Kolevzon, M. Pe'er, W. Wong, V. Shinder, L. Turnbull, C.B. Whitchurch, M. Elbaum, T.W. Gilberger, E. Yavin, J. Baum, R. Dzikowski, PfSec13 is an unusual chromatin-associated nucleoporin of Plasmodium falciparum that is essential for parasite proliferation in human erythrocytes, J. Cell. Sci. 126 (2013) 3055-3069. doi:10.1242/jcs.122119.

[17] L. Sheiner, J.L. Demerly, N. Poulsen, W.L. Beatty, O. Lucas, M.S. Behnke, M.W. White, B. Striepen, A systematic screen to discover and analyze apicoplast proteins identifies a conserved and essential protein import factor, PLoS Pathog. 7 (2011) e1002392. doi:10.1371/journal.ppat.1002392.

[18] F. Dzierszinski, M. Mortuaire, N. Dendouga, O. Popescu, S. Tomavo, Differential expression of two plantlike enolases with distinct enzymatic and antigenic properties during stage conversion of the protozoan parasite Toxoplasma gondii, J. Mol. Biol. 309 (2001) 1017-1027. doi:10.1006/jmbi.2001.4730.

[19] M. Gissot, R. Walker, S. Delhaye, L. Huot, D. Hot, S. Tomavo, Toxoplasma gondii chromodomain protein 1 binds to heterochromatin and colocalises with centromeres and telomeres at the nuclear periphery, PLoS ONE. 7 (2012) e32671. doi:10.1371/journal.pone.0032671.

[20] A. Olguin-Lamas, E. Madec, A. Hovasse, E. Werkmeister, I. Callebaut, C. Slomianny, S. Delhaye, T. Mouveaux, C. Schaeffer-Reiss, A. Van Dorsselaer, S. Tomavo, A novel Toxoplasma gondii nuclear factor TgNF3 is a dynamic chromatin-associated component, modulator of nucleolar architecture and parasite virulence, PLoS Pathog. 7 (2011) e1001328. doi:10.1371/journal.ppat.1001328.

[21] M. Gissot, R. Walker, S. Delhaye, T.D. Alayi, L. Huot, D. Hot, I. Callebaut, C. Schaeffer-Reiss, A.V. Dorsselaer, S. Tomavo, Toxoplasma gondii Alba proteins are involved in translational control of gene expression, J. Mol. Biol. 425 (2013) 1287-1301. doi:10.1016/j.jmb.2013.01.039.

[22] J. Thompson, In situ detection of RNA in blood- and mosquito-stage malaria parasites, Methods Mol. Med. 72 (2002) 225-233. doi:10.1385/1-59259-271-6:225.

[23] B. Shen, K.M. Brown, T.D. Lee, L.D. Sibley, Efficient gene disruption in diverse strains of Toxoplasma gondii using CRISPR/CAS9, MBio. 5 (2014) e01114-01114. doi:10.1128/mBio.01114-14.

[24] L. Miguet, G. Béchade, L. Fornecker, E. Zink, C. Felden, C. Gervais, R. Herbrecht, A. Van Dorsselaer, A. van Dorsselaer, L. Mauvieux, S. Sanglier-Cianferani, Proteomic analysis of malignant B-cell derived microparticles reveals CD148 as a potentially useful antigenic biomarker for mantle cell lymphoma diagnosis, J. Proteome Res. 8 (2009) 3346-3354. doi:10.1021/pr801102c.

[25] H. Li, R. Durbin, Fast and accurate short read alignment with Burrows-Wheeler transform, Bioinformatics. 25 (2009) 1754-1760. doi:10.1093/bioinformatics/btp324.

[26] D. Kim, G. Pertea, C. Trapnell, H. Pimentel, R. Kelley, S.L. Salzberg, TopHat2: accurate alignment of transcriptomes in the presence of insertions, deletions and gene fusions, Genome Biol. 14 (2013) R36. doi:10.1186/gb-2013-14-4-r36.

[27] B. Langmead, S.L. Salzberg, Fast gapped-read alignment with Bowtie 2, Nat. Methods. 9 (2012) 357-359. doi:10.1038/nmeth.1923.

[28] S. Anders, P.T. Pyl, W. Huber, HTSeq--a Python framework to work with high-throughput sequencing data, Bioinformatics. 31 (2015) 166-169. doi:10.1093/bioinformatics/btu638.

[29] R.C. Gentleman, V.J. Carey, D.M. Bates, B. Bolstad, M. Dettling, S. Dudoit, B. Ellis, L. Gautier, Y. Ge, J. Gentry, K. Hornik, T. Hothorn, W. Huber, S. Iacus, R. Irizarry, F. Leisch, C. Li, M. Maechler, A.J. Rossini, G. Sawitzki, C. Smith, G. Smyth, L. Tierney, J.Y.H. Yang, J. Zhang, Bioconductor: open software development for computational biology and bioinformatics, Genome Biol. 5 (2004) R80. doi:10.1186/gb-2004-5-10-r80.

[30] M.D. Robinson, D.J. McCarthy, G.K. Smyth, edgeR: a Bioconductor package for differential expression analysis of digital gene expression data, Bioinformatics. 26 (2010) 139-140. doi:10.1093/bioinformatics/btp616.

[31] S. Anders, W. Huber, Differential expression analysis for sequence count data, Genome Biol. 11 (2010) R106. doi:10.1186/gb-2010-11-10-r106. 
[32] K. Tamura, Y. Fukao, M. Iwamoto, T. Haraguchi, I. Hara-Nishimura, Identification and characterization of nuclear pore complex components in Arabidopsis thaliana, Plant Cell. 22 (2010) 4084-4097. doi:10.1105/tpc.110.079947.

[33] K. Tamura, I. Hara-Nishimura, The molecular architecture of the plant nuclear pore complex, J. Exp. Bot. 64 (2013) 823-832. doi:10.1093/jxb/ers258.

[34] B.M. Fontoura, G. Blobel, M.J. Matunis, A conserved biogenesis pathway for nucleoporins: proteolytic processing of a 186-kilodalton precursor generates Nup98 and the novel nucleoporin, Nup96, J. Cell Biol. 144 (1999) 1097-1112.

[35] D.J.P. Ferguson, S.F. Parmley, S. Tomavo, Evidence for nuclear localisation of two stage-specific isoenzymes of enolase in Toxoplasma gondii correlates with active parasite replication, Int. J. Parasitol. 32 (2002) 1399-1410.

[36] E.R. Griffis, S. Xu, M.A. Powers, Nup98 Localizes to Both Nuclear and Cytoplasmic Sides of the Nuclear Pore and Binds to Two Distinct Nucleoporin Subcomplexes, Mol. Biol. Cell. 14 (2003) 600-610. doi:10.1091/mbc.E02-09-0582.

[37] J. Enninga, A. Levay, B.M.A. Fontoura, Sec13 shuttles between the nucleus and the cytoplasm and stably interacts with Nup96 at the nuclear pore complex, Mol. Cell. Biol. 23 (2003) 7271-7284.

[38] L.A. Kelley, S. Mezulis, C.M. Yates, M.N. Wass, M.J.E. Sternberg, The Phyre2 web portal for protein modeling, prediction and analysis, Nat. Protocols. 10 (2015) 845-858. doi:10.1038/nprot.2015.053.

[39] C.E. Pritchard, M. Fornerod, L.H. Kasper, J.M. van Deursen, RAE1 is a shuttling mRNA export factor that binds to a GLEBS-like NUP98 motif at the nuclear pore complex through multiple domains, J. Cell Biol. 145 (1999) 237-254.

[40] J. Guizetti, R.M. Martins, S. Guadagnini, A. Claes, A. Scherf, Nuclear pores and perinuclear expression sites of var and ribosomal DNA genes correspond to physically distinct regions in Plasmodium falciparum, Eukaryotic Cell. 12 (2013) 697-702. doi:10.1128/EC.00023-13.

[41] H. Wang, H. Yang, C.S. Shivalila, M.M. Dawlaty, A.W. Cheng, F. Zhang, R. Jaenisch, One-step generation of mice carrying mutations in multiple genes by CRISPR/Cas-mediated genome engineering, Cell. 153 (2013) 910-918. doi:10.1016/j.cell.2013.04.025.

[42] J.A. DeGrasse, K.N. DuBois, D. Devos, T.N. Siegel, A. Sali, M.C. Field, M.P. Rout, B.T. Chait, Evidence for a shared nuclear pore complex architecture that is conserved from the last common eukaryotic ancestor, Mol. Cell Proteomics. 8 (2009) 2119-2130. doi:10.1074/mcp.M900038-MCP200.

[43] S.O. Obado, M. Brillantes, K. Uryu, W. Zhang, N.E. Ketaren, B.T. Chait, M.C. Field, M.P. Rout, Interactome Mapping Reveals the Evolutionary History of the Nuclear Pore Complex, PLOS Biol. 14 (2016) e1002365. doi:10.1371/journal.pbio.1002365.

[44] E. Fabre, W.C. Boelens, C. Wimmer, I.W. Mattaj, E.C. Hurt, Nup145p is required for nuclear export of mRNA and binds homopolymeric RNA in vitro via a novel conserved motif, Cell. 78 (1994) 275-289.

[45] T.C. Dockendorff, C.V. Heath, A.L. Goldstein, C.A. Snay, C.N. Cole, C-terminal truncations of the yeast nucleoporin Nup145p produce a rapid temperature-conditional mRNA export defect and alterations to nuclear structure, Mol. Cell. Biol. 17 (1997) 906-920.

[46] M.A. Powers, D.J. Forbes, J.E. Dahlberg, E. Lund, The vertebrate GLFG nucleoporin, Nup98, is an essential component of multiple RNA export pathways, J. Cell Biol. 136 (1997) 241-250.

[47] A. Radu, G. Blobel, M.S. Moore, Identification of a protein complex that is required for nuclear protein import and mediates docking of import substrate to distinct nucleoporins, Proc. Natl. Acad. Sci. U.S.A. 92 (1995) 1769-1773.

[48] A. Radu, M.S. Moore, G. Blobel, The peptide repeat domain of nucleoporin Nup98 functions as a docking site in transport across the nuclear pore complex, Cell. 81 (1995) 215-222.

[49] M.B. Frankel, L.J. Knoll, The ins and outs of nuclear trafficking: unusual aspects in apicomplexan parasites, DNA Cell Biol. 28 (2009) 277-284. doi:10.1089/dna.2009.0853.

[50] C.F. Brooks, M.E. Francia, M. Gissot, M.M. Croken, K. Kim, B. Striepen, Toxoplasma gondii sequesters centromeres to a specific nuclear region throughout the cell cycle, Proc. Natl. Acad. Sci. U.S.A. 108 (2011) 3767-3772. doi:10.1073/pnas.1006741108.

[51] C. Lemaittre, W.A. Bickmore, Chromatin at the nuclear periphery and the regulation of genome functions, Histochem. Cell Biol. 144 (2015) 111-122. doi:10.1007/s00418-015-1346-y.

[52] Y. Liang, M.W. Hetzer, Functional interactions between nucleoporins and chromatin, Curr. Opin. Cell Biol. 23 (2011) 65-70. doi:10.1016/j.ceb.2010.09.008.

[53] C.R. Brown, C.J. Kennedy, V.A. Delmar, D.J. Forbes, P.A. Silver, Global histone acetylation induces functional genomic reorganization at mammalian nuclear pore complexes, Genes Dev. 22 (2008) 627-639. doi:10.1101/gad.1632708.

[54] A. Bougdour, D. Maubon, P. Baldacci, P. Ortet, O. Bastien, A. Bouillon, J.-C. Barale, H. Pelloux, R. Ménard, M.-A. Hakimi, Drug inhibition of HDAC3 and epigenetic control of differentiation in Apicomplexa parasites, J. Exp. Med. 206 (2009) 953-966. doi:10.1084/jem.20082826. 
[55] D. Reinberg, R.J. Sims, de FACTo nucleosome dynamics, J. Biol. Chem. 281 (2006) 23297-23301. doi:10.1074/jbc.R600007200.

[56] C. Tous, A.G. Rondón, M. García-Rubio, C. González-Aguilera, R. Luna, A. Aguilera, A novel assay identifies transcript elongation roles for the Nup84 complex and RNA processing factors, EMBO J. 30 (2011) 1953-1964. doi:10.1038/emboj.2011.109.

[57] M. Costanzo, A. Baryshnikova, J. Bellay, Y. Kim, E.D. Spear, C.S. Sevier, H. Ding, J.L.Y. Koh, K. Toufighi, S. Mostafavi, J. Prinz, R.P. St Onge, B. VanderSluis, T. Makhnevych, F.J. Vizeacoumar, S. Alizadeh, S. Bahr, R.L. Brost, Y. Chen, M. Cokol, R. Deshpande, Z. Li, Z.-Y. Lin, W. Liang, M. Marback, J. Paw, B.-J. San Luis, E. Shuteriqi, A.H.Y. Tong, N. van Dyk, I.M. Wallace, J.A. Whitney, M.T. Weirauch, G. Zhong, H. Zhu, W.A. Houry, M. Brudno, S. Ragibizadeh, B. Papp, C. Pál, F.P. Roth, G. Giaever, C. Nislow, O.G. Troyanskaya, H. Bussey, G.D. Bader, A.-C. Gingras, Q.D. Morris, P.M. Kim, C.A. Kaiser, C.L. Myers, B.J. Andrews, C. Boone, The genetic landscape of a cell, Science. 327 (2010) 425-431. doi:10.1126/science.1180823.

[58] T. Burckin, R. Nagel, Y. Mandel-Gutfreund, L. Shiue, T.A. Clark, J.-L. Chong, T.-H. Chang, S. Squazzo, G. Hartzog, M. Ares, Exploring functional relationships between components of the gene expression machinery, Nat Struct Mol Biol. 12 (2005) 175-182. doi:10.1038/nsmb891.

[59] G.M. Hautbergue, M.-L. Hung, M.J. Walsh, A.P.L. Snijders, C.-T. Chang, R. Jones, C.P. Ponting, M.J. Dickman, S.A. Wilson, UIF, a New mRNA Export Adaptor that Works Together with REF/ALY, Requires FACT for Recruitment to mRNA, Current Biology. 19 (2009) 1918-1924. doi:10.1016/j.cub.2009.09.041.

\section{$\underline{\text { Captions }}$}

Fig. 1 Conditional knock-down of TgNup302 and phenotypic analysis. (a) Localization of the TgNup302 protein in the conditional knock-down strain (tagged at its $\mathrm{N}$ - and $\mathrm{C}$-termini) by confocal imaging using anti-HA (N-ter) and anti-Myc (C-ter) monoclonal antibodies under normal conditions (top) and after 48-hr ATc treatment (bottom). (b) TgNup302 double-tagged protein expression decreases under ATc treatment in the conditional knock-down strain. Immunoblots are shown for total protein extracts from the wild-type and TgNup302 iKD strain in the presence or absence of ATc for 24, 48, and $72 \mathrm{hrs}$. Western blots were probed with anti-HA (N-ter) and antiMyc (C-ter) antibodies. TgENO2 was probed as a loading control. (c) The appropriate level of TgNup302 expression is essential for parasite growth. Plaque assays were performed with 200 parasites of wild-type and TgNup302 iKD strains with or without ATc treatment for 7 days. The wild-type strain growth was not affected by ATc treatment (top). In contrast, the growth of the conditional TgNup302 knock-down strain was dramatically impaired in the presence of ATc. (d) Growth assay. Parasites from the parental (blue bars) or iKD TgNup302 iKD (red bars) strains were incubated with (crossed bars) or without ATc (dotted bars) for 48 hrs. The number of parasites per vacuole was scored for a minimum of 100 vacuoles. The average number of parasite is represented in this graph. The results shown are from three independent experiments. ${ }^{*}, \mathrm{P}<0.05 ; * *, \mathrm{P}<0.001$

Fig. 2 TgNup302 is involved in nuclear import and is essential for 18S RNA export. (a) TgNup302 is involved in the nuclear import of the TgENO2 protein. IFA was performed on paraformaldehyde-fixed intracellular parasites of the wild-type and TgNup302 iKD strains using a TgENO2 antibody ( $\alpha$-TgENO2) and staining of the nuclear DNA with DAPI. In the presence of ATc for $48 \mathrm{~h}$, the TgENO2 protein is mislocalized in the cytoplasm of TgNup302 iKD, whereas normal nuclear localization was observed in the absence of ATc for the parental and TgNup302 iKD strains, as expected. (b) TgENO2 localization in intracellular parasites. The percentage of parasites displaying nuclear and cytoplasmic TgENO2 signal was determined for 100 vacuoles in the parental and TgNup302 iKD strains with or without ATc for $48 \mathrm{~h}$ after IFA using a $\alpha$-TgENO2 staining. Predominant nuclear localization of TgENO2 was observed for the iKD strain without ATc and the parental parasites, whereas predominant cytoplasmic localization was observed for ATc-treated iKD parasites. The results shown are from three independent experiments. **, $\mathrm{P}<0.05$; ***, $\mathrm{P}<0.0001$. (c) TgNup302 is essential to 18S RNA export. RNA FISH was performed on intracellular parasites treated with or without ATc for $48 \mathrm{~h}$. Parasites of the parental and TgNup302 iKD strains were hybridized with Cy3-labeled 18S oligonucleotide primers (red), and the nuclear DNA was labelled with DAPI (blue). (d) 18S RNA localization in extracellular parasites. The quantity of nuclear and cytoplasmic Cy3-labeled $18 \mathrm{~S}$ signal for 100 parasites was determined for the parental and TgNup302 iKD strains with or without ATc for $48 \mathrm{~h}$. In the parental strain and iKD strains without ATc treatment, most 18S RNA is cytoplasmic, while it is mainly nuclear in the $\mathrm{iKD}$ parasites treated with ATc. The results shown are from three independent experiments. $* * *, \mathrm{P}<0.0001$

Fig. 3 TgNup302 depletion alters gene expression. (a) Total RNA purified from iKD TgNup302 parasites under either control conditions or ATc treatment for $48 \mathrm{hr}$ were analyzed by quantitative RT-PCR (dark bars). Genes 
coding for the transcripts identified to be upregulated with ATc treatment in RNA Seq were analyzed. Values are presented as $\log 2$ ratios of the signal given by the sample extracted from the ATc-treated parasites relative to those under control conditions (minus ATc). (b) Total RNA purified from iKD TgNup302 parasites under either control conditions or ATc treatment for $48 \mathrm{hr}$ were analyzed by quantitative RT-PCR. The TgNup302 gene was analyzed alongside the TgTubulin gene, which was not affected by ATc treatment. Values are presented as the Log2 ratio of ATc-treated parasites relative to those under control conditions (minus ATc).

Table1 Identification of proteins associated with NUP1 protein. Identification of proteins that were copurified from nuclear extract from the parental and TgNup1 iKD N-ter-HA Tag (in duplicate (1) and (2)) strains using nano LC-MS/MS. The affinity purification was performed under high stringency conditions and protein identity was determined using a composite target decoy database (50620 total entries) built with 3 strains of Toxoplasma gondii ToxoDB.org database (strains ME49, GT1 and VEG, release 12.0, September 2014, 25264 entries). Potential TgNup1 interacting proteins were identified and sorted by their secondary structure. FG repeats containing proteins are highlighted in green, alpha-solenoid protein in orange, beta-propeller in blue and coiled-coiled domains in yellow.

Fig. 4 Identified proteins interact with TgNup302. (a) Nuclear extract of $\sim 500 \times 10^{6}$ parasites for the TgFACT 140 (line 2), TgNup129 (line 3), TgNup134 (line 4) and TgNup407 (line 5) C-terminally myc tagged proteins in the TgNup302-HA iKD strain were immunoprecipitated using anti-myc antibodies. Extracts from the TgNup302 HA iKD strain were immunoprecipitated using anti-myc antibodies and used as negative controls. The immunoblot was probed with anti-HA antibody (upper panel) to detect interactions between the N-terminal domain of TgNup302 and the five different proteins. The same blots were reprobed with anti-myc antibodies to verify that the myc-tagged proteins were indeed immunoprcecipitated (bottom panel). The TgFACT140, TgNup129, TgNup134, TgNup407 C-terminally myc tagged proteins in the TgNup302-HA iKD strain and the TgNup302 HA iKD strain related inputs (produced from the same experiment) are shown in figure S9. (b) Nuclear extract of $\sim 300 \times 10^{6}$ parasites for the TgNup134 (positive control) and TgChromo1 (negative control) C-terminally myc tagged proteins in the TgNup302-HA iKD strain were immunoprecipitated using anti-Myc antibodies. Extracts from the TgNup302 HA iKD strain were immunoprecipitated using anti-Myc antibodies and used as negative controls. The immunoblot was probed with anti-HA (left panel) antibody to detect the TgNup302-HA protein in the input and eluates. The immunoblot was re-probed with an anti-myc antibody (right panel) to detect the myctagged proteins in the input and eluates.

Fig. 5 Identified proteins localize to the $T$. gondii nuclear pore. (a) Each potential partner was tagged using a Myc-tag in the TgNup302-HA iKD strain. Endogenous TgNup302 iKD was labelled with rabbit monoclonal antiHA (in red), and endogenous TgNup503, TgNup134, and TgNup115 were labelled with the mouse monoclonal anti-Myc (in green) antibody with or without ATc after $48 \mathrm{hr}$ of growth. (b) Measurement of cytoplasmic and nuclear Myc signals were determined for endogenous TgNup115 (vertical striped bars), TgNup134 (dotted bars) and TgNup503 (horizontal striped bars) potential partners without ATc treatment (blue) and after 48-hr ATc treatment (red). The ratio of the cytoplasmic signal versus nuclear signal was determined. The results shown are from three independent experiments. ${ }^{*}, \mathrm{P}<0.05$

Fig. 6 TgNup407 localization is perturbed in the TgNup302-deficient parasites. (a) TgNup407 protein was tagged using a Myc-tag in the TgNup302-HA iKD strain. Endogenous TgNup302 iKD was labelled with rabbit monoclonal anti-HA (in red) and endogenous TgNup407 was labelled with mouse monoclonal anti-Myc (in green) antibody with or without ATc after $48 \mathrm{hr}$ of growth. (b) Measurement of cytoplasmic and nuclear Myc signals were determined for endogenous TgNup503 (horizontal striped bars), TgNup407 (empty bars) potential partners without ATc treatment (blue) and after 48-hr ATc treatment (red). The ratio of the cytoplasmic signal versus nuclear signal was determined. The results shown are from three independent experiments. ***, $\mathrm{P}<0.0001$

Fig. 7 Codistribution of new nuclear pore proteins and TgNup302. (a) Codistribution between TgNup302-Nter HA-tag staining (in red) and Myc-tag staining (in green) for TgNup302 (C-terminus), TgNup67, TgNup129, TgNup503, TgNup115 and TgNup134 knock-ins. Values are measured with Imaris software from Structured Illumination Microscopy (SIM) (Pearson's coefficient in ROI volume). ***, $\mathrm{P}<0.0001$. (b) SIM images representing the codistribution of TgNup302 (C-ter), TgNup67, TgNup129, TgNup503, TgNup115 and TgNup134 proteins tagged using a Myc-tag (in green) in the TgNup302-HA (in red) iKD strain (highlighted by white arrows). (c) Number of pores per nucleus in TgNup302 (C-ter), TgNup67, TgNup129, TgNup503, TgNup115 and TgNup134 knock-ins. Values are measured with Imaris software from Structured Illumination Microscopy (SIM). ***, $\mathrm{P}<0.0001$ 
Fig. 8 CRISPR-Cas9 screening reveals the importance of the TgNup503 and TgNup134 proteins for the nuclear localization of TgENO2. The TgNup503 and TgNup134 proteins are important for the nuclear localization of the TgENO2 marker. IFA was performed on paraformaldehyde-fixed intracellular parasites of TgNup115, TgNup593, TgNup302, TgNup134, TgNup503, TgNup530, TgFACT140, TgNup67, TgNup37, TgNup68, TgNup216, TGGT1_228100, TgNup407, TgSec13, TgNup129 strains and TgAlba1 as a control using the TgENO2 antibody ( $\alpha$-TgENO2) and staining of the nuclear DNA with DAPI. The percentage of GFP-negative vacuoles with cytoplasmic TgENO2 and the percentage of GFP-positive vacuoles with cytoplasmic TgENO2 was determined. The results shown are from three independent experiments. ${ }^{* *}, \mathrm{P}<0.05$

Supporting information captions

Fig. S1 T. gondii TgNup302 is evolutionarily conserved among Eukaryota. Phylogenetic tree of $T$. gondii TgNup302 homologues based on ClustalW alignment of sequences identified by BLASTp searches using the entire sequence of TgTgNup302 gene against 15 apicomplexan parasites (brown), 5 Fungi (green), 3 Eumetazoa (blue) and 3 Plantae (purple). The tree was reconstructed by maximum likehood (ML) analyses with MEGA6 software. Five hundred bootstrap pseudo-replicates were used to give statistical support to the clades of the maximum likehood topology. Scale bar reflects number of substitutions per site. Numbers in the nodes of the tree reflect the percentage of bootstrap replicates supporting each node

Fig. S2 Construction of the TgNup302 iKD strain. (A) Schematic of the genetic approach used to produce the conditional knock-down strain by a promoter replacement strategy. After promoter replacement, the expression of TgNup302 is under the control of anhydrotetracyline (ATc). The TgNup302 gene was HA-tagged at its 5' (Top panel). (B) PCR was used to confirm the correct integration of the plasmid and the creation of the recombinant

944 locus using primer pairs $\mathrm{i}$ and ii (sequences are in TableS1 of supplementary data). (C) The double HA-tagged (N945 terminal) / Myc-tagged (C-terminal) strain for the TgNup302 gene was produced by introducing a Myc tag at the 9463 ' end of the gene

948 Fig. S3 PolyA+ RNA were mostly cytoplasmic in the parental and iKD strains. RNA FISH was performed on 949 intracellular parasites. Parasites of the parental and TgNup302 iKD strains were hybridized with Cy3-labeled 950 polyA+ oligonucleotides primers (red), and the nuclear DNA was labelled with DAPI (blue)

Fig. S4 The conditional expression of TgNup302 has no impact on TgChromo1 and TgNF3 localization.

953 Endogenous TgChromo1 and TgNF3 were labelled with the mouse monoclonal anti-Myc and 488nm Alexa goat 954 secondary antibody in parental and TgNup302 iKD strains with or without ATc treatment for 48hr 
956 Fig. S5 Ultrastructure of Toxoplasma gondii. Intracellular $T$. gondii tachyzoite showing the nucleus (N) for the parental RH $\Delta \mathrm{Ku} 80$ TaTi and iKD TgNup302 strains with or without ATc treatment. Bar=500nm

958

Fig. S6 Chromosomal distribution of genes differentially regulated in the TgNUP1 iKD strain. The chromosomal position and distribution of genes that were identified as differentially expressed, upregulated (top panel) and downregulated (bottom panel), in TgNup302 iKD after an ATc treatment for $48 \mathrm{hr}$

Fig. S7 Peptides identified for TgNup302. Peptides recovered from the immunoprecipitation of TgNup302 and mapped onto the TgNup302 sequence are highlighted in yellow. The autocatalytic domain sequence is underlined

Fig. S8 Predicted secondary structure features, fold and location for validated TgNups. The horizontal black line represents the polypeptide length of the proteins. The y axis indicates the confidence score of the predicted secondary structure element. Predicted $\alpha$-helices are indicated in blue, predicted $\beta$-sheets in orange, and predicted coiled coil regions are in red arrows. The green arrows indicate FG repeats.

970

Fig. S9 Identified proteins interact with TgNup302. (a) $5 \mu 1$ of nuclear extract (from $\sim 500 \times 10^{6}$ parasites) conserved before the immunoprecipitation (inputs) for the TgFACT140 (line 2), TgNup134 (line 3), TgNup129 (line 4) and TgNup407 (line 5) C-terminally myc tagged proteins in the TgNup302-HA iKD strain were analyzed by Western blot. The immunoblot was probed with anti-HA antibody to detect the presence of the TgNup302-HA protein in each strains before immunoprecipitation. (b) The immunoblot was reprobed with an anti-myc antibody to detect the myc tagged proteins: TgFACT140 (134kDa), TgNup129 (129kDa), TgNup134 (134kDa), TgNup407 $977 \quad(115 \mathrm{kDa})$.

Fig. S10 New components of the T. gondii nuclear pore. Each potential partners were tagged using a Myc-tag in the TgNup302-HA iKD strain. Endogenous TgNup302 iKD was labelled with the rabbit monoclonal anti-HA

981 (in red) and endogenous TgFACT140, TgNup129 were labelled with the mouse monoclonal anti-Myc (in green) antibody with or without ATc after $48 \mathrm{hr}$ of growth 
984 Fig. S11 Transfection efficiency for the Crisp-Cas9 screening. For each construction, the percentage of vacuole 985 with a positive GFP expression was monitored to determine the transfection efficiency at 24 hr after 986 electroporation, revealing that $\sim 30$ to $70 \%$ of cells received the plasmid. TgAlba1 is a negative control

987

988 Table S1: Oligonucleotides used in this study

989 Table S2: RNA-seq results. Presents all the genes up or down regulated with a FDR of 0.05

990 Table S3: Mass spectrometry results. The proteins highlighted in orange met the following criteria: less than 1

991 peptide in the control experiment and present in the two IP experiment

992 